\title{
Remote access to clinics: build back better
}

\author{
Clement Seeballuck, ${ }^{\star 1}$ Marc Beswick, ${ }^{2}$ Joseph Donnelly, ${ }^{3}$ Peter Mossey ${ }^{4}$ and Simon Shepherd ${ }^{5}$
}

\section{Key points}

A common challenge all trainers are experiencing - of interest to trainers and potential trainees. clinical engagement is becoming more commonplace and this is an area that dentists will need to explore.
Detailed thought processes mapped which could help develop a structure for other departments with similar issues.
May serve as a starting point for larger discussions on how to make national guidance.

\begin{abstract}
Since the advice to 'stay at home' on 23 March 2020, there has been an enforced upsurge in the use of remote services. Clinical constraints within the dental schools have drastically reduced trainee/patient exposure and utilising available tools to increase patient contact is invaluable. Developing robust protocols and frameworks for remote activities is key to ensuring safe, secure and effective learning experiences. In Dundee, we have invested in careful planning to implement online engagement with clinical activity. A collaborative and iterative approach with NHS Tayside and other key stakeholders has resulted in a safe, online, clinical protocol that trainees, trainers and patients can have confidence in. The purpose of this article is to share our experience and to open a dialogue to work synergistically with other institutions to contribute towards a UK-wide remote training strategy.
\end{abstract}

\section{Introduction}

In the current environment and as technology progresses, the value and potential applications of remote patient care have evolved. In recent years, there have been tremendous successes with telemedicine/teledentistry, such as NHS 24 and NHS 111. The 'hands on' nature of dentistry has perhaps slowed the adoption of remote clinical care compared with other disciplines, but the profession has still identified opportunities to adopt technology to enhance treatment delivery. Remote patient care can be considered as care undertaken using either telephones or online platforms (such as Near $\mathrm{Me}$, the online platform approved for use within NHS Scotland) ${ }^{1,2}$ and which facilitates access by reducing some of the traditional barriers to dental care, decreasing inequalities for

${ }^{1}$ Clinical Lecturer in Paediatric Dentistry, Dundee Dental School and Hospital, University of Dundee, UK; ${ }^{2}$ National Lead - Near Me Network, Technology Enabled Care Programme, Scottish Government, UK; ${ }^{3}$ Deputy Data Protection Officer, NHS Tayside, Dundee, UK; ${ }^{4}$ Consultant in Orthodontics and Associate Dean of Internationalisation, Dundee Dental School and Hospital, University of Dundee, UK; ${ }^{5}$ Consultant in Oral Surgery, Dundee Dental School and Hospital, University of Dundee, UK.

*Corresponding author: Clement Seeballuck Email address: c.seeballuck@dundee.ac.uk

Refereed Paper.

Accepted 7 April 2021

https://doi.org/10.1038/s41415-021-3714-4 those unable to travel. The associated reduced hospital footfall fulfils current COVID-19 criteria for distancing and reducing contact and liberates valuable resources for those procedures requiring a clinical environment. Multidisciplinary team collaborations are readily achievable with ease on a local, national and even international basis, offering clinicians in remote regions the benefit of distant expert advice. The potential pitfalls are, nonetheless, all too apparent. While potentially reducing one inequality, there is risk of introducing another for those in relative 'digital poverty', with digital illiteracy challenges or poor local connectivity.

With challenges to patient care arising from the global pandemic and consequent lockdowns there has been increasing need and use of remote services. Safe alternatives to the traditional clinical appointments have been explored and utilised. The reduced patient activity within teaching hospitals has naturally reduced trainee/patient exposure. Any potential solutions to increase patient contact without imposing on clinic accommodation is therefore invaluable. Online platforms may well prove part of the solution.

In line with this shift towards remote clinics, so too is there an increased need for remote training of our future colleagues. Virtual learning environments and online streaming platforms have enabled us to effectively deliver theoretical content to our trainees even during strict lockdown conditions and have become the 'new normal'.

However, how do we ensure that trainees get sufficient exposure to the clinical environment with limited opportunities to physically attend a clinic? Simulated training tools such as haptics and virtual reality are certainly exciting concepts but they lack the unpredictability and reality of a true patient interaction. These tools, while advancing rapidly, also still require specialist support and significant setup costs. Streaming live consultations from clinical environments directly to trainees in an appropriate remote location, although not an absolute substitute for physical clinics, can increase their exposure, allow for patient interaction and can form the essence of remote clinical training.

When using streaming platforms, it is crucial that we approach this in the appropriate way. Having transparent, comprehensive protocols and frameworks for remote activities is crucial for a safe, secure and supportive learning environment. Our work in Dundee to develop the necessary safe protocols has served to lay the foundations towards such a learning environment. A collaborative approach however with other key stakeholders will be necessary to further develop national guidance and ultimately build back better methods of clinical training. 
Fig. 1 Model for effective collaboration to develop remote training protocol

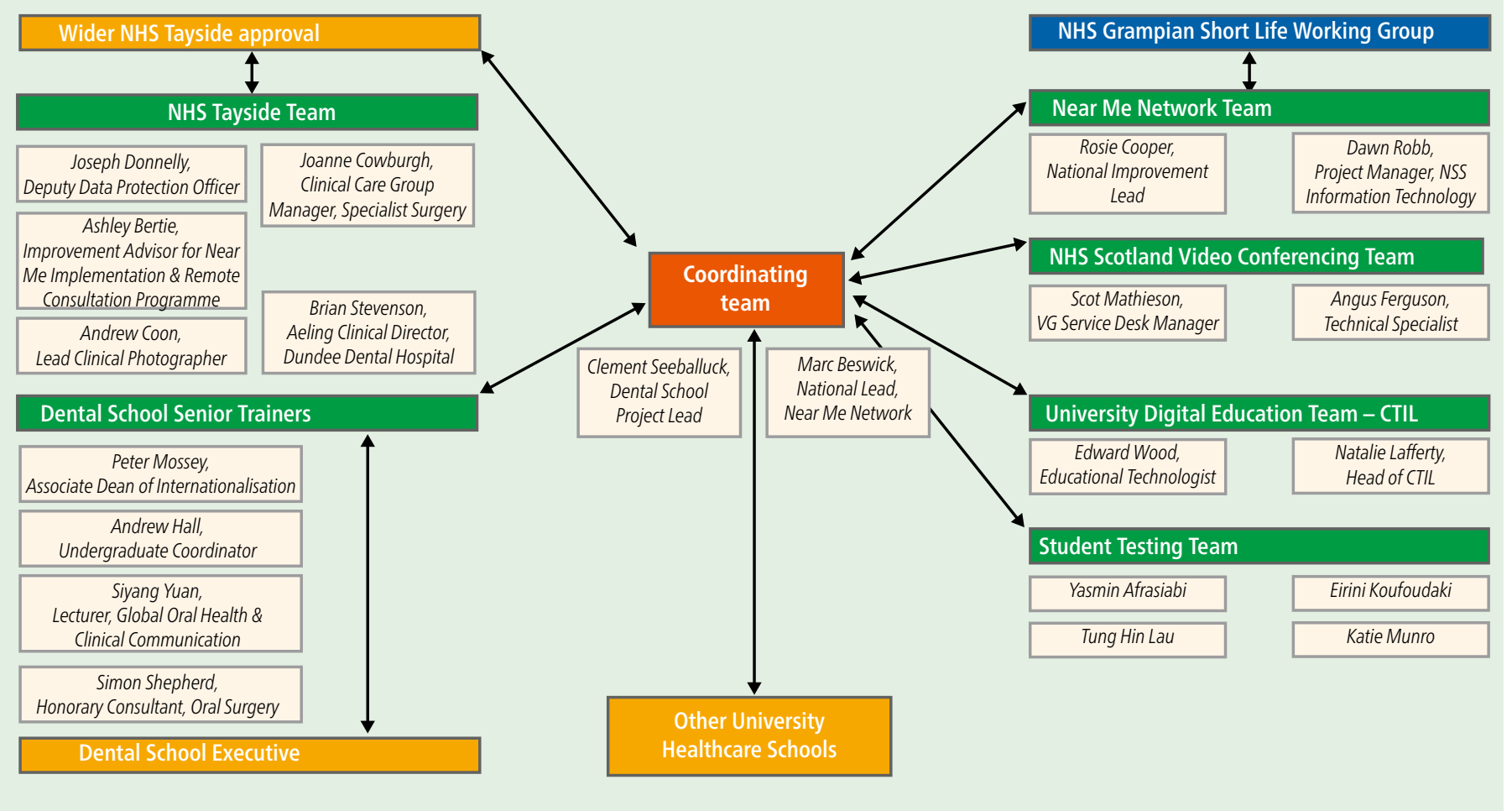

The purpose of this article is to share our experience in developing a protocol for remote clinical training, to open a dialogue and to work synergistically with other institutions to contribute towards a UK-wide remote training strategy. We will detail our approach including considerations and outline practical applications.

\section{Identifying the end users and the objectives of remote clinical training}

It is easy to identify the patient as an end user. The patient will always be the most important end user. As such, online platforms need to be optimised for the patient experience. A patient can, in essence, be any individual receiving care. This care can either be undertaken or observed remotely. Of course, the other end users will be both trainees undertaking clinical education, whether it be undergraduate or postgraduate and the trainers responsible for delivering the training. A trainee in one scenario may be a trainer in another, such as a postgraduate training an undergraduate.

The ultimate objectives of remote clinical training exercises are the same as those of clinical training exercises: increase patient exposure and gain clinical experience. The applications of remote training are numerous:

- End users isolating may still be able to attend a clinic in a safe manner: Anyone who has had to self-isolate will be aware of the disruption it causes. Having the opportunity for trainees to gain clinical experience while isolating may also provide 'inclusion' with their learning community at a difficult time. Similarly, a trainer may still be able to provide guidance and observation to advanced trainees from quarantine, rather than cancelling a clinic. This would also serve to protect patient care from disruption. Similar considerations apply for anyone advised to shield

- Remote clinical training can be used internationally. This will allow for enhanced clinical exposure for trainees undertaking an affiliated training programme overseas. Similarly, potential trainees considering enrolment may be able to participate in a session where appropriate. It must be noted that information sharing and data protection within the international setting will be a significant consideration. For this reason, we decided not to include this in our current protocol development

- Early-year trainees can safely engage in remote clinical training from a safe environment. Early-year undergraduates can safely observe a clinic, contextualising the theory elements of the course

- Remote clinical training can increase the number of trainees benefiting from a clinic. Remote trainees can observe a clinic in addition to trainees physically present
- Trainees from different professions can link into a clinic as part of a specific development module. For example, medical students could observe an oral cancer clinic.

The potential to readily form hierarchical learning communities within the sphere of remote clinical training is obvious. Advanced trainees can interact with patients with less experienced trainees observing, all being observed by experienced trainers. This opens opportunities for peer-assisted learning and feedback - crucial skills for trainees to develop.

Having identified our key teaching objectives, we needed to engage the local health board to jointly develop and approve appropriate protocols and pathways.

\section{Identifying key individuals who can work collaboratively to plan and create the necessary protocol for safe and effective implementation}

For this project to be a success, we needed to start with a sound foundation. Involving information governance and those experienced in online, patient-care platforms allowed us to address issues that we as clinicians may not have identified. The success of this project is a result of the engaged and enthusiastic team approaching this problem. Figure 1 shows the structure and members of the team along with 
the key roles they played. This model proved very successful in rapidly planning the project and resolving issues.

Regional collaborations with other health boards were fruitful through protocol sharing for the rapid development of documentation, a process which usefully identified a variety of differing scenarios for remote clinical training.

\section{Selecting an online platform for secure clinical streaming}

The platform needs to be:

- User-friendly - catering to a spectrum of trainee, trainer and patient digital literacy

- Secure - personal data of all parties concerned must be protected

- Supported - there should be technical support available; it should function on all operating systems and be accessible on university and NHS hardware

- Stable, reliable and with good image resolution - impact the learning experience

- Approved for patient interaction by the NHS and not amenable to recording

- Support multiple call entrants

- Functional on minimal hardware without niche specifications

- Minimal additional cost.

Table 1 demonstrates a selection of platforms considered. Based on these criteria, we determined Near Me to be the ideal choice.

Near $\mathrm{Me}$ is the patient video-consulting platform in NHS Scotland ${ }^{1,2}$ and is powered by a secure software called Attend Anywhere. It is web-based and does not require apps or special software. It was launched here in $2016^{3}$ with the national Technology-Enabled Care Programme, an initiative to increase the adoption of technology in health and care services. Near Me has been risk assessed and approved for remote consultations in NHS Scotland and was utilised within Allied Health Profession (AHP) undergraduate placements, enabling students to meet their learning outcomes. NHS Education for Scotland's AHP Practice Education team developed placement recovery guidance for Near Me use. ${ }^{4}$ This information helped inform early conversations around supporting remote dental training using Near Me.

\section{Protecting trainers, trainees and patients}

Arguably, this is the most controversial element when streaming to multiple end users. According to the General Dental Council guidance Preparing for practice, 5 'patient needs and protection are a priority in delivering education and training.

The use of such technology in healthcare settings brings new risks and responsibilities concerning personal data security.

Risks must be identified, assessed and mitigated to ensure compliance with data protection guidance and legislation.

Although the General Data Protection Regulation 2016 no longer applies in the UK after the end of the Brexit transition period, amendments made in the Data Protection, Privacy and Electronic Communications (Amendments etc) (EU Exit) Regulations 2019 and the Data Protection, Privacy and Electronic Communications (Amendments etc) (EU Exit) Regulations 2020 should ensure that the Data Protection Act 2018 remains aligned to ensure adequate data protection for the continued flow of data between the UK and the EU. ${ }^{6}$

There is clear guidance on remote clinician and patient interaction and on recording that interaction. However, the guidance on having a trainee present is less clear. The key difference between streaming a patient appointment rather than recording it is that potentially sensitive information is not retained. Therefore, issues regarding patient-identifiable information databases do not apply. Near Me cannot intrinsically record an appointment, a feature that protects clinical confidentiality.

When considering how to undertake a clinical interaction safely and securely, it is necessary to build into the process appropriate safeguards:

- Trainees should complete documented 'netiquette' and data protection training

- Clear guidance should be provided on the end-user environment

- Where possible, remote clinical engagement should take place on NHS or university equipment, also making it easier for eHealth technical support

- For errors or breaches in data, there needs to be an appropriate reporting pathway, such as the NHS Tayside Datix system

\begin{tabular}{|c|c|c|c|c|c|c|c|c|c|}
\hline Platform & User-friendly & Secure & Supported & Reliable & $\begin{array}{l}\text { Approved by } \\
\text { NHS Tayside }\end{array}$ & $\begin{array}{l}\text { Image sharing } \\
\text { capability }\end{array}$ & $\begin{array}{l}\text { Support } \\
\text { multiple call } \\
\text { entrants }\end{array}$ & Cost & $\begin{array}{l}\text { Recording } \\
\text { capability }\end{array}$ \\
\hline $\begin{array}{l}\text { Microsoft } \\
\text { Teams }\end{array}$ & $\begin{array}{l}\text { May prompt member } \\
\text { of public to download } \\
\text { an application } \\
\text { Can be complicated if } \\
\text { an individual already } \\
\text { has Teams }\end{array}$ & $\begin{array}{l}\text { Not assured for } \\
\text { patient data by } \\
\text { NHS Tayside }\end{array}$ & Yes & Yes & $\begin{array}{l}\text { Not for } \\
\text { patient- } \\
\text { identifiable } \\
\text { information* }\end{array}$ & Yes & Yes & $\begin{array}{l}\text { Already used } \\
\text { by university } \\
\text { and NHS }\end{array}$ & Yes $^{*}$ \\
\hline Zoom & Yes & $\begin{array}{l}\text { Not assured for } \\
\text { patient data by } \\
\text { NHS Tayside* }\end{array}$ & Yes & Yes & $\mathrm{No}^{*}$ & Yes & Yes & $\begin{array}{l}\text { Requires } \\
\text { minimal } \\
\text { subscription }\end{array}$ & Yes $^{*}$ \\
\hline $\begin{array}{l}\text { Blackboard } \\
\text { Collaborate }\end{array}$ & $\begin{array}{l}\text { Less intuitive } \\
\text { Requires access to } \\
\text { Blackboard }\end{array}$ & $\begin{array}{l}\text { Not assessed } \\
\text { by NHS Tayside* }\end{array}$ & $\begin{array}{l}\text { Supported for } \\
\text { Blackboard } \\
\text { Ultra users }\end{array}$ & Yes & $\mathrm{No}^{*}$ & Yes & Yes & $\begin{array}{l}\text { Already used } \\
\text { by university }\end{array}$ & Yes $^{*}$ \\
\hline Near Me & Yes & Yes & Yes & Yes & Yes & Yes & $\begin{array}{l}\text { Stable up to } \\
\text { approx. } 4 \\
\text { callers }\end{array}$ & $\begin{array}{l}\text { Already in } \\
\text { use by NHS } \\
\text { Tayside }\end{array}$ & $\begin{array}{l}\text { Not from } \\
\text { within the } \\
\text { platform }\end{array}$ \\
\hline Skype & Yes & $\begin{array}{l}\text { Not assured for } \\
\text { patient data by } \\
\text { NHS Tayside* }\end{array}$ & Yes & Yes & $\mathrm{No}^{*}$ & Yes & Yes & $\begin{array}{l}\text { Most } \\
\text { functions } \\
\text { free to use }\end{array}$ & Yes $^{*}$ \\
\hline
\end{tabular}




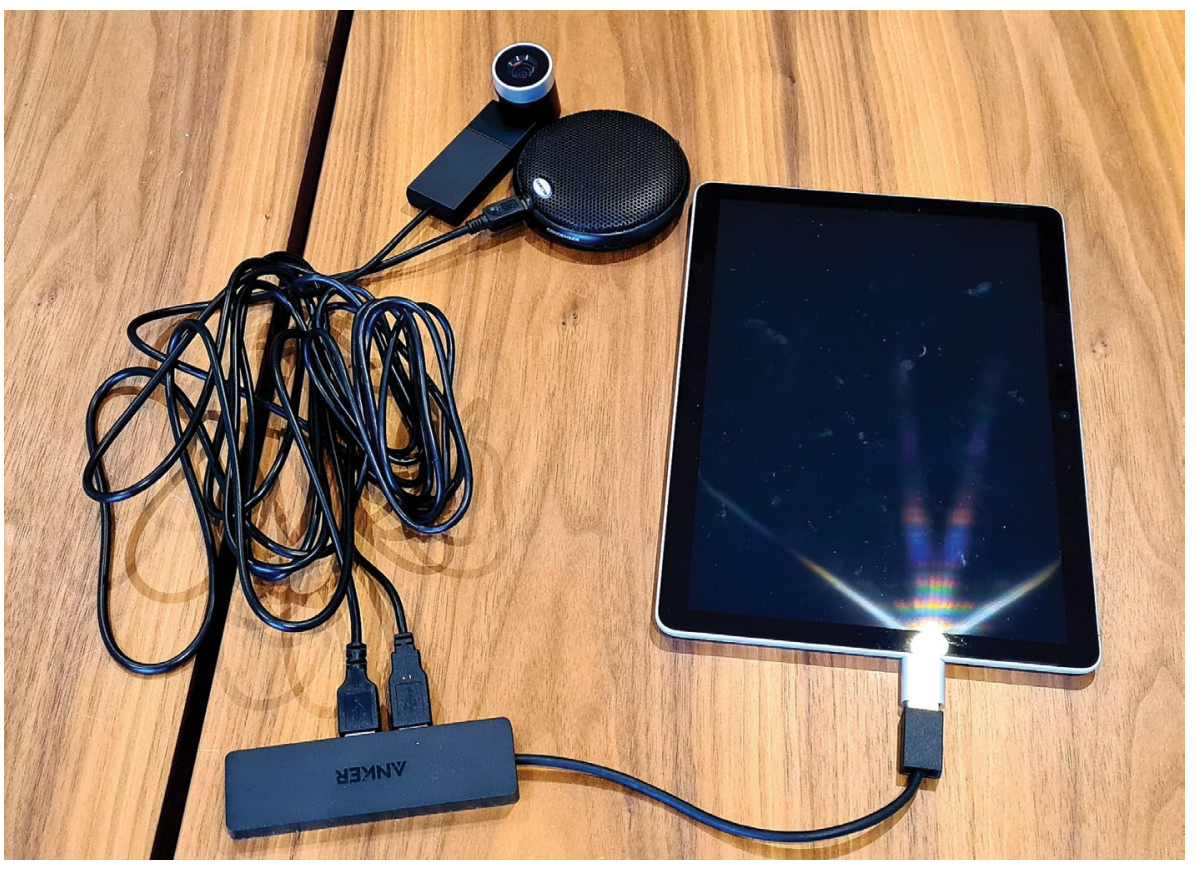

Fig. 2 Simple, mobile, clinical camera set-up

- Clear guidance should be provided regarding clinical note taking by trainees, appointment bookings and outcome processes

- Backup measures should be in place to ensure care is not impacted by IT, trainee or trainer issues.

\section{Deciding what is achievable}

Do we investigate the available technology first, then plan exercises? Or do we first consider ideal training activities and then seek technological solutions? Understanding the range of available technology is important but needs to be considered alongside your budget.

Choices will then be refined based on planned teaching. The learning outcomes outlined in Preparing for practice $e^{5}$ served as a good foundation when planning scenarios for process mapping, with most of these applicable to remote clinical training provided two-way communication is possible. There is also a need to teach skills that prepare trainees for delivering digital services and indeed Preparing for practice recognises trainers need to include 'technological and clinical advances'. While it is acknowledged that hands-on skills cannot readily be taught remotely, students and trainees can, through observing an experienced clinician, become familiar with techniques, learn communication skills and better develop verbal interactions and interventions, such as providing advice, obtaining consent and discussing treatment plans.

We felt the following scenarios capture a spectrum of learning exercises:

1. Trainee in remote location. Clinician undertaking clinical care in clinical environment

2. Trainee undertaking clinical care in clinical environment. Clinician observing remotely

3. Trainee in same location as clinician, undertaking remote clinical consultation

4. Trainee in remote location. Clinician undertaking remote clinical consultation.

Except for scenario two, training exercises can be observational or interactive.

\section{Considering hardware and end user interaction}

It is important to consider the technical needs of the end user:

- Patients may have limited equipment, poor connectivity and minimal digital literacy. Most interactions will have low-quality webcams or front-facing phone cameras. Intraoral views may be of limited quality

- Trainees - undergraduates in particular may lack the latest technology/advanced IT skills. The possibility of providing additional equipment (eg high-resolution monitors, perhaps for viewing images) may depend on whether it is a postgraduate course with three trainees, or an undergraduate course with 100
- Clinical-setting end users may require access to high-end equipment, so we need to consider:

o What views are required? A webcam can monitor a trainee or allow them to observe communication but is unable to offer intraoral assessment

o What about set-ups for specific purposes such as operator, microscope, or lightmounted cameras for closer intraoral or detailed procedural views?

o Does the system need to be wired or wireless? Transmitting images wirelessly can cause degradation in quality or a time lag. Although tablets and phones are useful, Android and iOS systems do not easily support external cameras. For wired systems, consider the logistics of power outlets and cabling

o 'Off the shelf' or custom made? Custombuilt systems may be unproven and unreliable, time consuming and more prone to quality control issues. Larger companies buy in bulk and commit to research and development, so may be more economical and predictable

o Cross infection - it is essential that setups conform to regulations.

We are currently trialling wired and 'semi-wired' set-ups, whereby a camera and microphone are wired to a tablet that supports Windows and is connected to the school network wirelessly. Figure 2 shows the portable configuration we are using for oral medicine clinics. This is modular, with a range of different camera and microphone options compatible to suit different clinical objectives.

We are confident a configuration of two cameras in the clinical environment will work for most scenarios, depending on the objectives of the exercises.

\section{Conclusion}

Remote clinical engagement has potential for improving training of our future colleagues at undergraduate and postgraduate levels, increasing trainee exposure to a clinical environment with minimal safety considerations. To future-proof training and develop fail-safes for the unexpected, we need to embrace technological advances and create robust adaptable protocols. In challenging times, there is a clear need for alternative training strategies. 
We have made progress in developing strategies by adopting a synergistic, collaborative approach. Our user agreement document has now been given approval by the local health board and by sharing this documentation we will be able to support other training institutions within the health board area to initiate their own remote clinical training more smoothly, without repeating the same challenging process. Patient care is a universal goal for all of our professions, after all.

Our next challenge is to test equipment solutions to optimise the experience. Recently, in the Dundee Dental School, students have remotely attended in numbers not possible in the clinical setting. Live oral medicine clinics and the feedback has been very positive, with students appreciating the efforts in such challenging times. There will certainly be setbacks in exploring potential camera combinations and configurations. However, with an open approach whereby these setbacks and successes are shared, we hope to minimise the replication of the same mistakes across institutions.

Undoubtedly, there are numerous similar ventures across the UK. We are all facing the same challenges and perhaps seeking similar solutions. Our intention was to share our experience, to open dialogue and to improve clinical training opportunities for us all. The way forward in the COVID-19 and post-COVID era is likely closer collaborations and consolidation of the development of a UK-wide strategy.

There is strength in numbers. The merits of remote patient care as an adjunct to traditional care will ensure its relevance and integration into practices beyond the pandemic.

\section{Ethics declarations}

The authors declare that there are no conflicts of interest.

Author contributions

Clement Seeballuck: lead author. Marc Beswick: contributing author regarding Near Me and online platforms. Joseph Donnelly: contributing author. regarding information governance and data protection. Peter Mossey: contributing author to main body of text. Simon Shepherd: contributing author to main body of text.

\section{References}

1. Scottish Government. What is Near Me? 2020. Available at https://www.nearme.scot (accessed January 2021).

2. NHS Scotland Video Conferencing Service. About Near Me. 2021. Available at https://www.vc.scot.nhs.uk/ near-me/ (accessed January 2021).

3. Scottish Government. Attend Anywhere/Near Me video consulting service evaluation 2019-2020: report. 2017. Available at https://www.gov.scot/publications/ evaluation-attend-anywhere-near-video-consultingservice-scotland-2019-20-main-report/pages/2/ (accessed January 2021).

4. NHS Education for Scotland. Covid 19 Placement Recovery. 2019. Available at http://www.knowledge. scot.nhs.uk/ahppe/covid-19-placement-recovery.aspx (accessed January 2021).

5. General Dental Council. Preparing for Practice: Dental team learning outcomes for registration (2015 revised edition). 2015. Available at https://www.gdc-uk.org/ docs/default-source/quality-assurance/preparing-forpractice-(revised-2015).pdf (accessed November 2021)

6. The European Commission. Adequacy decisions: How the EU determines if a non-EU country has an adequate level of data protection. Available at https://ec.europa. eu/info/law/law-topic/data-protection/internationaldimension-data-protection/adequacy-decisions en (accessed November 2021). 